\title{
Heavy Quarkonium Mass Spectra in A Coulomb Field Plus Quadratic Potential Using Nikiforov-Uvarov Method
}

\author{
Ahmed Al-Jamel ${ }^{1} \&$ Hatem Widyan $^{1}$ \\ ${ }^{1}$ Physics Department, Al Al-Bayt University, Mafraq, Jordan \\ Correspondence: Ahmed Al-Jamel, Department of Physics, Al Al-Bayt University, Mafraq 25113, Jordan. Tel: \\ 962-2-629-7000-3592. E-mail: aaljamel@gmail.com, aaljamel@aabu.edu.jo
}

Received: May 30, 2012 Accepted: June 16, 2012 Online Published: July 18, 2012

doi:10.5539/apr.v4n3p94 URL: http://dx.doi.org/10.5539/apr.v4n3p94

\begin{abstract}
In this work, the spin-averaged mass spectra of heavy quarkonia $(c \bar{c}$ and $b \bar{b})$ in a Coulomb plus quadratic potential is studied within the framework of nonrelativistic Schrodinger equation. The energy eigenvalues and eigenfunctions are obtained in compact forms for any $l$-value using Nikiforov-Uvarov method. The obtained results are used to produce potential parameters $(a, b$, and $\delta)$ for the charmonium and bottomonium systems, from which then their full mass spectra are determined. The obtained values are compared with the available experimental results. The predictions from our model are found to be in good agreement with the experimental results. As a side result, the Hydrogen atom known spectrum is recovered.
\end{abstract}

Keywords: heavy quarkonium, mass spectra, Nikiforov-Uvarov

\section{Introduction}

Since their discoveries, investigation of heavy quarkonium systems $(c \bar{c}, b \bar{b}, c \bar{b}, b \bar{c})$ provides us with a crucial role for quantitative tests of QCD and the standard model. For detailed review of heavy quarkonium physics recent progress, see e.g. (Brambilla \& Vario, 2007; Brambilla et al., 2005; Zalewski, 1998) and the references therein. Because of the heavy masses of the constituent quarks $\left(m_{q}>>\Lambda_{Q C D}\right.$, i.e. than about $200 \mathrm{MeV}$, where $\Lambda_{Q C D}$ is the hadronization scale), many features of these systems can be studied within the framework of nonrelativistic Schrodinger equation, where one assumes that the quark-antiquark strong interaction is described by a phenomenological potential. There are many potential models that are commonly used to study heavy quarkonium spectra; for instance, Martin, logarithmic, and Cornell potentials (Al-Jamel, 2011; Patel \& Vinodkumar, 2009; Rai, Patel, \& Vinodkumar, 2008; Reyes, Rigol, \& Soneira, 2003; Zalewski, 1998). Any of these potential should take into account the two distinctive features of the strong interaction, namely, asymptotic freedom and confinement. A successful potential model for such systems is the one that produces its mass spectra in agreeing with the experimental data within about $20 \mathrm{MeV}$ and leptonic decay widths within a factor of two (Bhanot \& Rudaz, 1978).

The main obstacle in such studies arises due to the abscence of the exact solutions of Schrödinger equation for such systems, particulalry when the centrifugal potential $\frac{\hbar^{2} l(l+1)}{2 \mu r^{2}}$ is included. For $l \neq 0$, there are some approximation techniques, analytical and numerical, were developed, such as supersymmetry (Morales, 2004), $1 / N$ expansion (Bag, Panja, \& Dutt, 1992), Pekeris approximation (Pekeris, 1934), variational methods (Montgomery, 2001, 2011), and asymptotic iteration methods (Ciftci, Hall, \& Saad, 2009).

In the present paper, we consider the mass spectra of heavy quarkonium systems in a Coulomb plus quardatic potential using Nikiforov-Uvarov method. In section 2, we review the main formalism of the conventional Nikiforov-Uvarov (NU) method used in our analysis. In section 3, we present our main problem and its analytic solution. In section 4, results and discussion are given. In the last section, summary and conclusions are presented.

\section{Nikiforov-Uvarov Method}

The Nikiforov-Uvarov method (hereafter, NU) is a method that provides us an exact solution of non-relativistic Schrödinger equation, or Schrödinger-like equation, for certain shape of potentials. It is based on the solutions of general second order linear differential equation with special orthogonal functions (Nikiforov \& Uvarov, 1988; Szego, 1975). With an appropriate $s=s(x)$ coordinate transformation, the Schrödinger equation in one 
dimension for certain potentials is reduced to a generalized equation of hypergeometric type of the form

$$
\psi^{\prime \prime}(s)+\frac{\tilde{\tau}(s)}{\sigma(s)} \psi^{\prime}(s)+\frac{\widetilde{\sigma}(s)}{\sigma^{2}(s)} \psi(s)=0
$$

where $\sigma(s)$ and $\widetilde{\sigma}(s)$ are polynomials, at most second-degree, and $\tilde{\tau}(s)$ is a first-degree polynomial. For a careful derivation of $\mathrm{Eq}(1)$, one can refer to (Nikiforov \& Uvarov, 1988). To find the particular solution of Eq.(1), we use the following transformation

$$
\psi(s)=\phi(s) y(s)
$$

which reduces Eq.(1) to an equation of hypergeometric type,

$$
\sigma(s) y^{\prime \prime}+\tau(s) y^{\prime}+\lambda y=0,
$$

where $\phi(s)$ satisfies

$$
\phi^{\prime}(s) / \phi(s)=\pi(s) / \sigma(s),
$$

and $y(s)$ is the hypergeometric type function whose polynomial solutions satisfies the Rodrigues relation

$$
y_{n}(s)=\frac{B_{n}}{\rho(s)} \frac{d^{n}}{d s^{n}}\left[\sigma^{n}(s) \rho(s)\right] .
$$

In the above equation, $B_{n}$ is the normalization constant and $\rho$ is called the weight function and must satisfy the condition

$$
(\sigma \rho)^{\prime}=\tau \rho .
$$

The function $\pi$ and the parameter $\lambda$ required for this method are defined as

$$
\pi=\frac{\sigma^{\prime}-\tilde{\tau}}{2} \pm \sqrt{\left(\frac{\sigma^{\prime}-\tilde{\tau}}{2}\right)^{2}-\widetilde{\sigma}+k \sigma}
$$

and

$$
\lambda=k+\pi^{\prime}
$$

Here, $\pi(s)$ is a polynomial with the parameter $s$ and the determination of $k$ is crucial in the calculation of $\pi(s)$. To determine the value of $k$, the expression under the square root in Eq.(7) must be square of a polynomial. Hence, the follwoing new eigenvalue equation for the Schrödinger equation will be established

$$
\lambda=\lambda_{n}=-n \tau^{\prime}-\frac{n(n-1)}{2} \sigma^{\prime \prime},(n=0,1,2, \ldots)
$$

where

$$
\tau(s)=\tilde{\tau}(s)+2 \pi(s)
$$

and it will have a negative derivative. By equating Eq.(8) with Eq.(9), we obtain the energy eigenvalues.

\section{Formulation of the Problem And Its Analytic Solutions}

As a phenomenological model for a heavy quarkonium system $(q \bar{q})$, we consider the following spin-averaged potential

$$
V(r)=-\frac{a}{r}+b r^{2}
$$

where $a$ and $b$ are non-negative constants and $r$ is the interquark distance. This potential has two parts: The first is $-a / r$, which corresponds to the potential induced by one-gluon exchange between the quark and its anti-quark that dominated at short distances, while the second part $b r^{2}$ accounts for quark confinement at large distances. The nonrelativistic radial Schrödinger equation for this system is

$$
\frac{-1}{2 \mu r^{2}} \frac{d}{d r}\left(r^{2} \frac{d R(r)}{d r}\right)+\left(V(r)+\frac{l(l+1)}{2 r^{2}}\right) R(r)=E R(r),
$$

subject to the boundary condition $R(\infty)=0$. Using $u(r)=r R(r)$, the radial Schrödinger equation is 
transformed to

$$
\frac{d^{2} u(r)}{d r^{2}}+2 \mu\left(E+\frac{a}{r}-b r^{2}-\frac{l(l+1)}{2 r^{2}}\right) u(r)=0 .
$$

Making the change of variable $x=1 / r$, this equation then becomes:

$$
\frac{d^{2} u(x)}{d x^{2}}+\frac{2 x}{x^{2}} \frac{d u(x)}{d x}+\frac{2 \mu}{x^{4}}\left(E+a x-\frac{b}{x^{2}}-\frac{\gamma}{2} x^{2}\right) u(x)=0 .
$$

where $\gamma=l(l+1)$. Next,we propose the following approximation scheme on the term $\frac{b}{x^{2}}$. Let us assume that there is a characteristic radius $r_{0}$ of the meson. Then the scheme is based on the expansion of $\frac{b}{x^{2}}$ in a power series around $r_{0}$, i.e. around $\delta \equiv \frac{1}{r_{0}}$, in the $x$-space, up to the second order, so that the $b$-dependent term, preserves the original form of Eq.(14) as if the term $\frac{b}{x^{2}}$ were not exist. This is similar to Pekeris approximation, which helps to deform the centrifugal potential such that the modified potential can be solved by NU method. Setting $y=(x-\delta)$ and around $y=0$ it can be expanded into a series of powers as

$$
\begin{gathered}
\frac{b}{x^{2}}=\frac{b}{(\delta+y)^{2}} \approx \frac{b}{\delta^{2}}\left(1-2 \frac{y}{\delta}+3 \frac{y^{2}}{\delta^{2}}\right) \\
=\frac{b}{\delta^{4}}\left(6 \delta^{2}-8 \delta x+3 x^{2}\right) .
\end{gathered}
$$

Note that, within this approximation, an extra model parameter is introduced, viz., $\delta$. It should be mentioned that the choice $b=0$ eliminates $\delta$ from the calculations, and the effect of the confining quadratic potential dissappears and then we recover the free Coulombic field problems. Substituting this into the radial Schrödinger equation, we obtain

$$
\frac{d^{2} u(x)}{d x^{2}}+\frac{2 x}{x^{2}} \frac{d u(x)}{d x}+\frac{2}{x^{4}}\left(-A+B x-C x^{2}\right) u(x)=0,
$$

where $A=\mu\left(-E+\frac{6 b}{\delta^{2}}\right), B=\mu\left(a+\frac{8 b}{\delta^{3}}\right)$ and $C=\mu\left(\frac{\gamma}{2}+\frac{3 b}{\delta^{4}}\right)$. By comparing this last equation with Eq.(1) we have $\tilde{\tau}=2 x, \sigma=x^{2}, \widetilde{\sigma}=2\left(-A+B x-C x^{2}\right)$, and so we can apply the NU method. Therefore

$$
\pi= \pm \sqrt{(k+2 C) x^{2}-2 B x+2 A} .
$$

The constant $k$ is chosen such as the function under the square root has a double zero, i.e. its discrimenant $\Delta=4 B^{2}-8 A(k+2 C)=0$. Therefore,

$$
\pi= \pm \frac{1}{\sqrt{2 A}}(2 A-B x)
$$

Thus,

$$
\tau=2 x \pm \frac{2}{\sqrt{2 A}}(2 A-B x) .
$$

For bound state solutions, we choose the positive sign in the above equation so that the derivative

$$
\tau^{\prime}=2-\frac{2 B}{\sqrt{2 A}}
$$

can have negative values depending on the values of parameters $A$ and $B$, which in turn depend on the potential parameters $a, b$ and $\delta$. Therefore, from Eq.(20), and the definitions of $A$ and $B$, we have

$$
E<\frac{6 b}{\delta^{2}} \text { and } B>\sqrt{2 A}
$$

which sets a lower limit on the mean radius reciprocal $\delta$ for a given energy value and the constants $a$ and $b$. Using Eq.(8), then we have 


$$
\lambda=\frac{B^{2}}{2 A}-2 C-\frac{B}{\sqrt{2 A}} .
$$

On the other hand, from Eq.(9)

$$
\lambda_{n}=-n\left(2-\frac{2 B}{2 \sqrt{A}}\right)-n(n-1)
$$

Comparing Eq.(8) and Eq.(9), one gets $A$, from which one can then obtain the energy eigenvalues as

$$
E_{n l}=\frac{6 b}{\delta^{2}}-\frac{2 \mu\left(a+\frac{8 b}{\delta^{3}}\right)^{2}}{\left[(2 n+1) \pm \sqrt{1+4 l(l+1)+\frac{24 \mu b}{\delta^{4}}}\right]^{2}} .
$$

The eigenfunctions in this approximation scheme can then be determined as discussed in section 2. Using Eq.(4), one obtains:

$$
\phi(x)=x^{-\frac{B}{\sqrt{2 A}}} e^{\frac{-\sqrt{2 A}}{x}}
$$

and from Eq.(6) we obtain the weight function

$$
\rho(x)=x^{-\frac{2 B}{\sqrt{2 A}}} e^{\frac{-2 \sqrt{2 A}}{x}}
$$

Thus,

$$
y_{n}(x)=B_{n} x^{\frac{2 B}{\sqrt{2 A}}} e^{\frac{2 \sqrt{2 A}}{x}} \frac{d^{n}}{d x^{n}}\left(x^{2 n} x^{-\frac{2 B}{\sqrt{2 A}}} e^{\frac{-2 \sqrt{2 A}}{x}}\right)
$$

The corresponding $u_{n l}(x)$ wave functions are then found to be

$$
u_{n l}(x)=N_{n l} x^{\frac{B}{\sqrt{2 A}}} e^{\frac{\sqrt{2 A}}{x}} \frac{d^{n}}{d x^{n}}\left(x^{2 n} x^{-\frac{2 B}{\sqrt{2 A}}} e^{\frac{-2 \sqrt{2 A}}{x}}\right)
$$

where $N_{n l}$ is the normalization constant determined by arguing that $\int_{0}^{\infty}\left|u_{n l}(r)\right|^{2} d r=1$. By setting $x=1 / r$, and using that $R_{n l}(r)=\frac{u_{n l}(r)}{r}$, then we obtain

$$
R_{n l}(r)=N_{n l} r^{-\frac{B}{\sqrt{2 A}}-1} e^{\sqrt{2 A} r}\left(-r^{2} \frac{d}{d r}\right)^{n}\left(r^{-2 n+\frac{2 B}{\sqrt{2 A}}} e^{-2 \sqrt{2 A} r}\right)
$$

\section{Results and Discussion}

In this section, we use the obtained energy eigenvalue formula to study the mass spectra of heavy quarkonia. To be specific, we consider the charmonium $(c \bar{c})$ and bottomonium $(b \bar{b})$. Before we proceed our analysis, and as a side result, we use our results to recover the Coulomic field problem, for instance the H-atom known spectrum. As clear from Eq. (24), the energy $E_{n l}$ depends on the potential parameters $a$ and $b$, and $\delta$. Its formula contains two choices in the denominator, \pm . The true choice can be fixed by retreiving the free hydrogen-atom energy spectrum, which can be done by setting $b=0$, and $a=1$. In this case, the known H-atom spectrum and wavefunctions are recovered by interpreting the integer $n(=0,1,2, \ldots)$ that appears in our calculations above as not the principal quantum number, $\tilde{n}=1,2,3, \ldots$, for the hydrogenic problem, but rather through the relation

$$
\tilde{n}=n+l+1 \text {. }
$$

In this case, using Eq. (8-44) of (Gasiorowicz 2003), one can see that positive sign in the denominator of Eq.(24) should be chosen. Thus, the H-atom spectrum in Hartree units is:

$$
E_{n l}=-\frac{1}{2(n+l+1)^{2}}
$$

which is in agreement with the free $\mathrm{H}$ atom spectrum known in literatures. 
Table 1 . The spin-averaged mass spectrum for $c \bar{c}$ (in $\mathrm{GeV}$ ) with $a=3.171, b=0.095$, and $\delta=0.6$. The experimental data are taken from (Nakamura et al., 2010).

\begin{tabular}{lcl}
\hline State & Present & Experiment \\
$1 \mathrm{~S}$ & 3.096 & 3.096 \\
$1 \mathrm{P}$ & 3.433 & - \\
$2 \mathrm{~S}$ & 3.686 & 3.686 \\
$1 \mathrm{D}$ & 3.767 & 3.770 \\
$2 \mathrm{P}$ & 3.910 & - \\
$3 \mathrm{~S}$ & 3.984 & 4.040 \\
$4 \mathrm{~S}$ & 4.150 & 4.263 \\
\hline
\end{tabular}

Table 2. The spin-averaged mass spectrum for $b \bar{b}$ (in $\mathrm{GeV}$ ) with $a=1.697, b=0.384$, and $\delta=1.3$. The experimental data are taken from (Nakamura et al., 2010)

\begin{tabular}{lcc}
\hline State & Present & Experiment \\
$1 \mathrm{~S}$ & 9.460 & 9.460 \\
$1 \mathrm{P}$ & 9.840 & 9.900 \\
2S & 10.023 & 10.023 \\
$1 \mathrm{D}$ & 10.140 & 10.161 \\
2P & 10.160 & 10.260 \\
3S & 10.280 & 10.355 \\
$4 \mathrm{~S}$ & 10.420 & 10.580 \\
\hline
\end{tabular}

We next examine the possibility of producing the mass spectra using

$$
M(q \bar{q})=2 m_{q}+E
$$

where $E$ is given by Eq. (24). Using $m_{c}=1.488 \mathrm{GeV}$ and $m_{b}=4.686 \mathrm{GeV}$ and from the experimental $1 S$ state mass values $M_{c \bar{c}}(1 S)=3.096 \mathrm{GeV}$ and $M_{b \bar{b}}(1 S)=9.460 \mathrm{GeV}$ one can determine the potential parameters $a, b$, and $\delta$. Then, the rest of the mass spectra are determined using Eq.(32). The results are shown in Tables 1 and 2. The obtained spin-avergaed mass values are found to differ by no more than $1.5 \%$ for charmonium and by $1.3 \%$ for bottomonium in comparison with the expermintal data except for the $4 \mathrm{~S}$ charmonium state. As a nonrelativistic model, this agreement with the experimental results is fair. It should be mentioned that fine and hyperfine structure of heavy quarkonium mass spectra where the spin plays a major role can be taken into account if we use relativisitic corrections and the approperiate relativistic Schrodinger equation.

\section{Conclusions}

In this work, the spin-averaged mass spectra of heavy quarkonia $(c \bar{c}$ and $b \bar{b})$ in a Coulomb plus quadratic potential were studied within the framework of nonrelativistic Schrodinger equation. The energy eigenvalues and eigenfunctions were obtained analytically for any $l$-value using Nikiforov-Uvarov method. Using the available experimental data, the obtained energy formula were used fit charmonium and bottomonium mass spectra from which then the potential parameters $(a, b$, and $\delta)$ were determined. These parameters were then used to reproduce the mass spectra, which then were compared with the experimental results. The predictions from our model are found to be in good agreement with the experimental results. As a side result, the Hydrogen atom known spectrum is recovered.

\section{Acknowledgment}

We would like to thank Prof. Eqab Rabei from our department and M. F. Al-Jamal from mathematical sciences department at Michigan Technological University for their fruitful suggestions.

\section{References}

Al-Jamel, A. (2011). Heavy quarkonia with Cornell potential on noncommutative space. Journal of Theoretical and Applied Physics, 5(1), 21-24. 
Bag, M., Panja, M. M., \& Dutt, R. (1992). Modified shifted large- $N$ approach to the Morse oscillator. Phys. Rev. A, 46, 9. http://d x.doi.org/10.1103/PhysRevA.46.6059

Bhanot, G., \& Rudaz, S. (1978). A new potential for quarkonium. Phys. Lett., 78B, 119. http://dx.doi.org/10.1016/0370-2693(78)90362-3.

Brambilla, N., \& Vario, A. (2007). Heavy Quarkonium Physics-Theoretical Status. Acta. Phys. Polon. B, 38, 3429-3440.

Brambilla, N. et al. (2005). Heavy Quarkonium Physics. hep-ph/0412158 CERN-2005-005. -Geneva: CERN, 487.

Ciftci, H., Hall, R. L., \& Saad, N. (2009). Study of a confined hydrogen-like atom by the asymptotic iteration method. International Journal of Quantum Chemistry, 109, 931-93. http://dx.doi.org/10.1002/qua.21905

Gasiorowicz, S. (2003). Quantum Physics (3rd Ed.). New york, NY: John Wiley \& Sons, Inc.

Montgomery, H. E., Jr. (2001). Variational Perturbation Theory of the Confined Hydrogen Atom. Int. J. Mol. Sci., 2, 103-108. http://dx.doi.org/10.3390/i2020103

Montgomery, H. E., Jr. (2011). Variational perturbation treatment of the confined hydrogen atom. Eur. J. Phys., 32, 1275. http://dx.doi.org/10.1088/0143-0807/32/5/015

Morales, D. A. (2004). Supersymmetric improvement of the Pekeris approximation for the rotating Morse potential. Chem. Phys. Letters, 394, 68. http://dx.doi.org/10.1016/j.cplett.2004.06.109.

Nakamura, K. et al. (2010). (Particle Data Group). J. Phys. G, 37, 075021.

Nikiforov, A. F., \& Uvarov, V. B. (1988). Special Functions of Mathematical Physics (Birkhauser, Basel).

Patel, B., \& Vinodkumar, P. C. (2009). Properties of $(Q \bar{Q})(Q \in c, b)$, mesons in Coulomb plus Power potential. J. Phys. G., 36, 035003.

Pekeris, C. L. (1934). The Rotation-Vibration Coupling in Diatomic Molecules. Phys. Rev., 45, 98. http://dx.doi.org/10.1103/PhysRev.45.98

Rai, A. J., Patel, B., \& Vinodkumar, P. C. (2008). Properties of $Q \bar{Q}$ mesons in non-relativistic QCD formalism. Phys. Rev. C, 78, 055202.

Reyes, E. C., Rigol, M., \& Soneira, J. R. (2003). Hadron Spectra from a Non-Relativistic Model with Confining Harmonic Potential. Revista Brasileira de Ensino de F'isica, 25(1).

Szego, G. (1975). Orthogonal Polynomials. American Mathematical Society, Providence, Rhode Island (4th Ed.).

Zalewski, K. (1998). Nonrelativistic Description of Heavy Quarkonia. Acta Phys. Polon. B, 29, 2535-2538. 